\title{
Intravitreal anti-vascular endothelial growth factor injection combined with panretinal photocoagulation for neovascular glaucoma in Indonesian patients with diabetes mellitus: a prospective study
}

Widya Artini, ${ }^{1}$ Ajeng Gracia, ${ }^{1}$ Aria Kekalih, ${ }^{2}$ Virna Dwi Oktariana, ${ }^{1}$ Andi Arus Victor, ${ }^{1}$ Anna Puspitasari Bani ${ }^{1}$

pISSN: 0853-1773 • elSSN: 2252-8083 https://doi.org/10.13181/mji.v28i3.2865 Med J Indones. 2019;28:258-67

Received: July 24, 2018

Accepted: June 18, 2019

Authors' affiliations: 'Department of Ophthalmology, Faculty of Medicine, Universitas Indonesia, Jakarta, Indonesia, ${ }^{2}$ Department of Community Medicine, Faculty of Medicine, Universitas Indonesia, Jakarta, Indonesia

\section{Corresponding author:}

Widya Artini

Department of Ophthalmology, Faculty of Medicine, Universitas Indonesia, Cipto Mangunkusumo Kirana Hospital, Jalan Kimia No. 8, Central Jakarta 10320, DKI Jakarta, Indonesia

Tel/Fax: +62-21-31902885

E-mail: ikkesumantri@gmail.com

\begin{abstract}
BACKGROUND The aim of this study was to determine the levels of vascular endothelial growth factor (VEGF) in the aqueous humor and the effect of intravitreal anti-VEGF injection combined with panretinal photocoagulation (PRP) on the management in diabetes mellitus (DM) patients with neovascular glaucoma (NVG).
\end{abstract}

METHODS This study was a prospective, interventional study in DM patients with NVG. Paracentesis followed by intravitreal bevacizumab (IVB) injection was performed in all eyes. The concentration of VEGF obtained from paracentesis was measured. In week-1, the intraocular pressure (IOP), sectorial iris neovascularization (NVI), and visual acuity were documented, and management was continued with PRP laser over a period of 1 week. All parameters and additional interventions performed after PRP were also recorded.

RESULTS A total of 18 eyes from 17 patients were studied. The mean (SD) level of VEGF in the aqueous humor was $3,864(1,468) \mathrm{pg} / \mathrm{ml}$, and the mean (SD) of initial IOP was $39(10.2) \mathrm{mmHg}$. There was a significant reduction in IOP in week-1 after the first intervention to $24.4(8.0) \mathrm{mmHg}(p=0.001)$; however, at 2 weeks the IOP increased to $30.4(6.7) \mathrm{mmHg}$. NVI showed significant regression in week-1 after IVB combined with PRP laser $(p<0.05)$. All eyes required additional glaucoma implants (14 eyes) and cyclocryotheraphy (4 eyes).

CONCLUSIONS In the eyes of diabetes patients with NVG, VEGF levels were high. With the use of IVB, the IOP was reduced, and NVI regressed; however, due to the severe stages of disease, all eyes required glaucoma surgery.

KEYWORDS Ahmed glaucoma valve, bevacizumab, intraocular pressure, neovascularization, photocoagulation, vascular endothelial growth factor
Indonesia has the fifth highest prevalence of adult onset diabetes mellitus (DM) worldwide, and the majority of patients with diabetes are unaware of their disease. ${ }^{1}$ Diabetic retinopathy may cause permanent blindness; therefore, early diagnosis and prompt treatment are essential. Moreover, the disease has been associated with structural and functional changes in the retinal microvasculature, including basal membrane thickening, pericytes and endothelial damage, cellular capillary development, microaneurysm, blood flow changes, leukocyte adhesion, and hyperpermeability, which may result in neovascularization. ${ }^{2,3}$ Vascular endothelial growth factor (VEGF) is an angiogenic factor that is responsible for triggering the proliferative capillary of endothelial cells in the iris. ${ }^{4}$ The mechanism is induced by ischemia, hypoxia, and oxidative stress in the retina as a result of uncontrolled DM. In the advanced stages of proliferative diabetic retinopathy, levels of VEGF in the vitreous are estimated to be three times higher than those in the normal eye.5,6 Prolonged ischemia in the retina may cause severe 
complications, including neovascular glaucoma (NVG), which is one of the most difficult types of glaucoma to treat. ${ }^{7-10}$ In NVG, levels of VEGF in the aqueous humor are increased, whereas under normal circumstances (the normal range is: mean [SD] $=59.5$ (24.6) $\mathrm{pg} / \mathrm{ml}$ ), VEGF in the aqueous humor is known to regulate microvascular endothelial permeability and the permeability of Schlemm's canal endothelium, essential for conventional aqueous humor outflow. ${ }^{11}$

The management of NVG has not yet been standardized; moreover, glaucoma and retinal eye specialists differ in their respective management

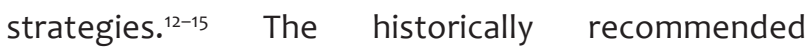
treatment for NVG is panretinal photocoagulation (PRP) laser treatment, despite the fact that this measure takes time to eliminate neovascularization in the iris and retina, as PRP laser treatment requires a few weeks for the initial effect to take place. Intravitreal anti-VEGF injection is believed to offer more rapid relief and is preferred by most retinal eye specialists as the first line of treatment for a vascular abnormality. ${ }^{12}$ Meanwhile, glaucoma specialists opt for anti-VEGF injection combined with a glaucoma drainage implant to address high intraocular pressure (IOP).13,15 The combination of paracentesis and intravitreal anti-VEGF injection, followed by PRP laser treatment, as a first line management strategy will achieve a better result and eliminate the need for more invasive glaucoma surgery. The aim of this study was to describe the levels of VEGF and the role of intravitreal anti-VEGF injection combined with PRP in the management course of NVG in the eyes of patients with DM.

\section{METHODS}

This study was a prospective interventional case series study conducted in Cipto Mangunkusumo Hospital, Jakarta, Indonesia. All patients signed an informed consent form before intervention was performed. The Ethics Committee of the Faculty of Medicine, Universitas Indonesia also approved the study (No. 1092/UN2.F1/etik/2015) in accordance with the principles of the Helsinki Declaration.

\section{Subjects}

All patients included in the study had DM and NVG. The inclusion criteria were patients with DM aged $\geq 30$ years who had uncontrolled glaucoma (IOP
$>25 \mathrm{mmHg}$ ) despite maximal medical therapy with iris or anterior chamber angle neovascularization within a sectorial range of 2 to 4 and who were willing to participate in this study. The patients who met any of the following criteria: no light perception; NVG with a cause other than DM; contraindications for IVB; and a history of cerebrovascular disease (stroke), cardiovascular disease (heart disease or heart attack), prolonged coagulation parameters, and previous glaucoma surgeries were excluded.

\section{First intervention}

All subjects underwent general health and eye examinations, including routine preoperative bloodwork, fasting, and 2-hour postprandial blood glucose. The initial assessment included visual acuity (VA) measurement using the International Standard Visual Acuity Chart, which was then converted to IogMAR, and IOP measured with a Goldmann applanation tonometer. A comprehensive eye examination was also performed, which included anterior segment evaluation using a slit lamp, degree of neovascularization of the iris (NVI), anterior chamber angle, gonioscopy, and posterior chamber evaluation. Under low light conditions, eyes underwent gonioscopy in the primary position using a Sussman-style 4-mirror goniolens (Ocular instrument, Inc., USA) with dynamic measurement. When the cornea was too hazy due to high IOP, gonioscopy was implemented after paracentesis. All angles were assessed with a narrow beam of light, the vertical beam of which was used to measure the superior and inferior angles. Indentation gonioscopy was carried out to detect any degree of synechial fibrovascular or neovascularization. Four angles of the anterior chamber were documented in the medical records, together with the structure of the angle, neovascularization, peripheral anterior synechiae, and degree of pigment. To determine the degree of neovascularization, the iris were divided into four quadrants and, by using slit lamp rubeosis, the iris were detected and captured in ImageJ (taken at least three times). The final calculation was performed by two experts (glaucoma consultants).

The paracentesis were performed with a small operating microscope under sterile conditions in the outpatient clinic. After antiseptic preparation of the local area, the anterior chamber at temporal limbus was punctured at the 3 o'clock position 
(right eye) or 9 o'clock position (left eye) using a 1cc syringe with a 30-Gauge needle parallel to the iris plane. A total of $150-200 \mu \mathrm{l}$ of aqueous humor was drawn to lower the IOP. Afterwards, a single dose of $0.05 \mathrm{ml} / 1.25 \mathrm{mg}$ bevacizumab was administered intravitreally (3-4 $\mathrm{mm}$ from the corneal limbus), called intravitreal bevacimuzab (IVB), by glaucoma consultants to neutralize the VEGF concentration in the vitreous. Bevacizumab (Avastin ${ }^{\circledR}$, Genentech, Inc., San Francisco, CA) is a full-length humanized murine monoclonal antibody that works through non-specific binding of all VEGF isoforms, thereby acting as an anti-VEGF substance. After procedure was done, antibiotic eye drops was given 4 times a day for 5 days.

Patients were evaluated on day-1, day-7, and at month-1 after IVB. Measurements were taken of VA, IOP, the sectorial degree of NVI surface, and the anterior chamber angle. Conventional PRP lasers (532 nm wavelength laser, Visulas ${ }^{\circledR}$, Zeiss, Germany) were used on all eyes as soon as posterior segment visibility was ensured, within a period of 7 days. The combination of IVB and PRP laser administration within the first 7 days of the intervention was termed the first intervention.

\section{Second intervention}

If IOP remained above $22 \mathrm{mmHg}$ for 1 month after the first intervention despite maximal anti-glaucoma medication, glaucoma surgery was then implemented as rescue therapy and termed the secondary intervention. The type of surgery was chosen on the basis of remaining VA. In the case of no VA or light perception, cyclocryo photocoagulation was the preferred surgical intervention. In cases with VA of at least $1 / 300$, Ahmed implant surgery was performed. Follow-up was carried out at 1, 3, and 6 months after surgery with measurement of the IOP and VA and documentation of other interventions, if any.

\section{Glaucoma Ahmed implant technique}

Ahmed implant surgery ${ }^{16}$ was performed in all subjects by 2 glaucoma surgeons (WA, VDO) under local (peribulbar injection) or general anesthesia. A single-plate Ahmed glaucoma implant (184 mm²; New World Medical, Rancho Cucamonga, California, USA) was used. A limbal-based conjunctival incision at the supra-temporal quadrant was performed. The plate implants were then pushed under the conjunctiva flap posteriorly and sutured to the sclera $8 \mathrm{~mm}$ behind the limbus using 10-0 nylon sutures. A paracentesis was made, and a viscoelastic was inserted into the anterior chamber. The end point of the long silicon tube was cut bevel-up approximately 1-2 $\mathrm{mm}$ in length at the anterior chamber from the limbus. Using a 23-G needle, the anterior chamber is then punctured 1-2 $\mathrm{mm}$ posteriorly to the limbus. The long tube, which was still located above the sclera, was fixed using 10-0 nylon sutures, covered with donor sclera in order to prevent its exposure, and fixed to the sclera using Vicryl ${ }^{\mathrm{TM}}$ 8-0 sutures. Conjunctival and sub-Tenon flaps were then sutured with Vicryl ${ }^{\mathrm{TM}}$ 8-0. After the procedure was completed,

Table 1. Subject characteristics

\begin{tabular}{|c|c|}
\hline Variables & $\mathrm{n}(\%)$ \\
\hline Number of subjects & 17 \\
\hline Number of eyes & 18 \\
\hline \multicolumn{2}{|l|}{ Gender } \\
\hline Male & $10(58.8)$ \\
\hline Female & $7(41.2)$ \\
\hline Age (years), mean (SD) & $54.3(13.7)$ \\
\hline \multicolumn{2}{|l|}{ Systemic disease } \\
\hline DM & $17(94.4)$ \\
\hline Hypertension + DM & $8(44.4)$ \\
\hline \multicolumn{2}{|l|}{ Previous treatment } \\
\hline Anti-glaucoma agents & $18(100)$ \\
\hline Panretinal photocoagulation laser & $8(44.4)$ \\
\hline $\begin{array}{l}\text { Blood glucose level (mg/dl), } \\
\text { mean (SD) }\end{array}$ & $237.8(13.7)$ \\
\hline Baseline VA (LogMAR), mean (SD) & $1.5(0.19)$ \\
\hline $\begin{array}{l}\text { Baseline intraocular pressure }(\mathrm{mmHg}) \text {, } \\
\text { mean (SD) }\end{array}$ & $39(10.2)$ \\
\hline $\begin{array}{l}\text { Degree of iris neovascularization } \\
\text { (quadrant), mean (SD) }\end{array}$ & $3.43(0.20)$ \\
\hline $\begin{array}{l}\text { VEGF level before IVB }(\mathrm{pg} / \mathrm{ml}) \text {, mean } \\
\text { (SE) }\end{array}$ & $6,015(1,468)$ \\
\hline $\begin{array}{l}\text { VEGF level before IVB }(\mathrm{pg} / \mathrm{ml}) \text {, mean } \\
\text { (SE) }\end{array}$ & $8,488(2,451)$ \\
\hline $\begin{array}{l}\text { VEGF level before IVB (pg/ml), mean } \\
\text { (SE) (non-PRP) }\end{array}$ & $2,924(1,606)$ \\
\hline \multicolumn{2}{|l|}{ Second intervention } \\
\hline Ahmed implant & $14(77.7)$ \\
\hline Cyclocryoteraphy & $4(22.2)$ \\
\hline
\end{tabular}

$\mathrm{SD}=$ standard deviation; $\mathrm{DM}=$ diabetes mellitus; $\mathrm{VA}=\mathrm{visual}$ acuity; VEGF=vascular endothelial growth factor; IVB=intravitreal bevacizumab; $\mathrm{SE}=$ standard of error; $\mathrm{PRP}=$ panretinal photocoagulation 
antibiotic and steroid eye drops were administered for 2 months.

\section{Cryodestructive surgery technique}

All subjects underwent this procedure under local anesthesia (retrobulbar injection and ketarolac intra vena injection). A retinal cryomachine (Keeler, USA) was used under sterile conditions in the operating theater. The cryoprobe was cooled to approximately $-80^{\circ} \mathrm{C}$ for $45 \mathrm{sec}$, producing an ice ball following the procedure, and the probe removed when the ice had thawed. Six applications on average were performed in 3 quadrants of the globes over the conjunctiva 2-3 $\mathrm{mm}$ behind the limbus by avoiding the 3, 6, and 9 o'clock positions. On completion of the procedure, antibiotic and steroid eye pointments were administered for 2 weeks. ${ }^{16}$

\section{Measurement of VEGF concentrations in the aqueous humor}

The aqueous humor taken during paracentesis was immediately stored in microcentrifuge tubes, transported using containers packed in dry ice, and maintained at $-80^{\circ} \mathrm{C}$ until analysis. The concentration of VEGF in the aqueous humor was measured by using sandwich enzyme-linked immune-sorbent assay (ELISA) (Quantikine ${ }^{\circledR}$ VEGF ELISA Kit; R\&D Systems) with a Varioskan ${ }^{\mathrm{TM}}$ reader (Thermo Scientific ${ }^{\mathrm{TM}}$ ) in the Laboratory of the Department of Biochemistry, Faculty of Medicine, Universitas Indonesia.

\section{Data analysis}

All data were analyzed using SPSS software, version 20 (IBM). Regression of quadrants had NVI was shown in proportional bar chart. Wilcoxon signed-rank tests were performed to compare numerical data before and after intervention. Spearman correlation was used to analyze correlations between 2 variables. A $p$-value of $<0.05$ indicated that the difference was statistically significant with a power of $80 \%$.

\section{RESULTS}

A total of 18 eyes with NVG from 17 patients with DM and proliferative diabetic retinopathy (PDR) were enrolled in this study. Subject characteristics are shown in Table 1. All eyes needed additional glaucoma intervention; Ahmed implant surgery was used in 14 eyes and cyclocryo photocoagulation in 4 eyes. Meanwhile, subgroup analysis revealed that the mean (SD) aqueous humor level of VEGF in the PRP and non-PRP subgroups prior to IVB administration was $8,488(2,451) \mathrm{pg} / \mathrm{ml}$ versus $2,924(1,606) \mathrm{pg} / \mathrm{ml}$ ( $p$ $=0.183$ ) (Figure 1).

As shown in Figure 2, 14/18 eyes (71.4\%) with NVG had NVI in all 4 quadrants at baseline, and on day-1 post IVB, only 7/18 eyes (35.7\%) had NVI in 4 quadrants. On day-7, none of the eyes had NVI in 4 quadrants, 2 eyes had the maximum number of $\mathrm{NVI}$ in only 2 quadrants, and there was no sign of neovascularization in $15 / 18(64.3 \%)$ eyes.

\section{The outcome after paracentesis followed by IVB injection and combined with PRP}

There was significant reduction of IOP after the first intervention on day-7 from 39 (10.2) to 24.4 (8.02) ( $\Delta=14.6 \mathrm{mmHg} ; p=0.001)$. However, up to day-30 the mean (SD) of IOP remained above the normal value (30.4 [6.7] $\mathrm{mmHg}$ ) (Figure 3). Afterward, second interventions were needed in all eyes.

\section{Second intervention}

All eyes needed additional glaucoma intervention, and the mean (SD) of intial IOP before the second intervention was 30.4 (6.7) $\mathrm{mmHg}$; the mean (SD) of IOP after the second intervention was 21.5 (12) $\mathrm{mmHg}$

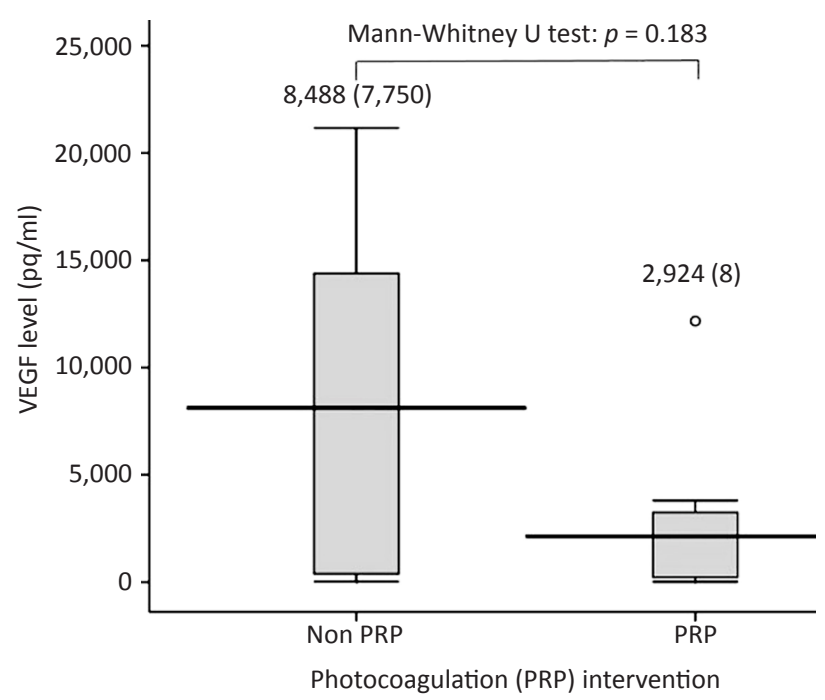

Figure 1. The level of VEGF in the aqueous humor in PRP and non-PRP diabetic NVG eye subgroups before IVB injection. $\mathrm{VEGF}=$ vascular endothelial growth factor; PRP=panretinal photocoagulation; NVG=neovascular glaucoma; IVB=intravitreal bevacizumab 


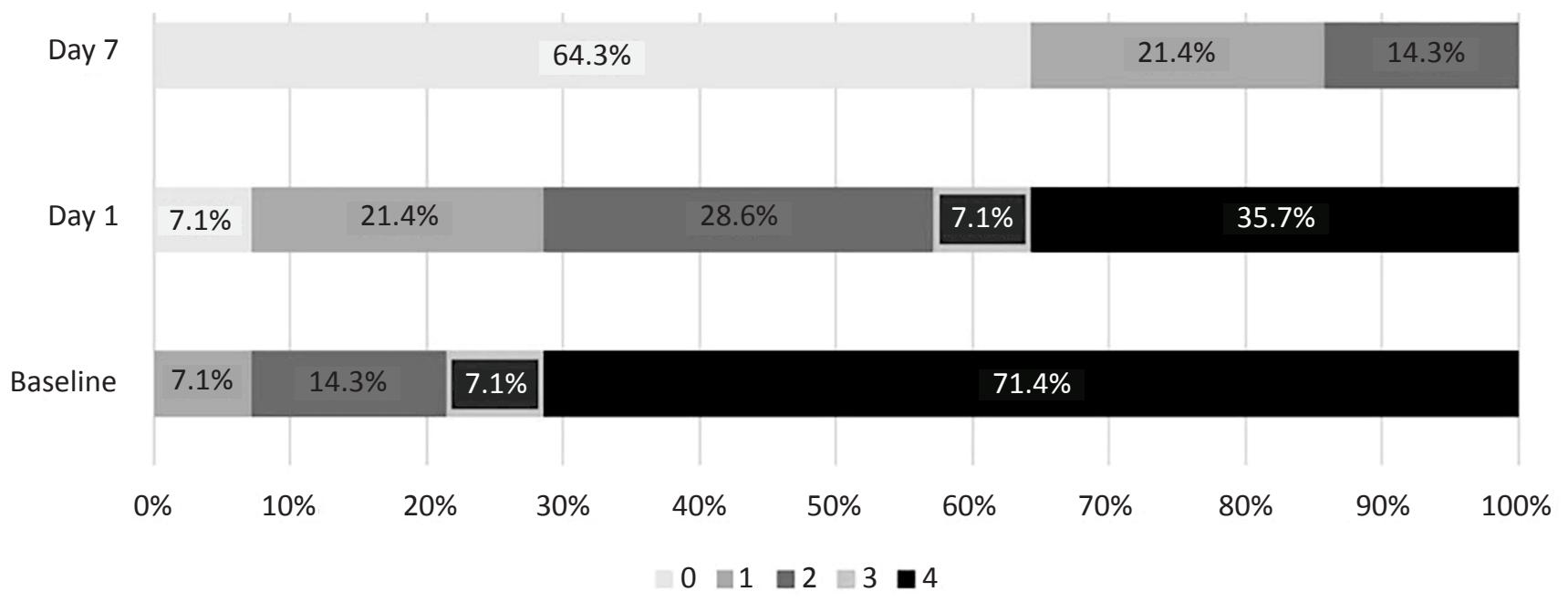

Figure 2. Degree of neovascularization of the iris in baseline, day-1 to day-7 before and after intravitreal bevacizumab injection. $0=$ no neovascularization; $1=$ neovascular in 1 quadrant of iris; $2=$ neovascular in 2 quadrants iris; $3=$ neovascular in 3 quadrants iris; 4=neovascular in covering all iris

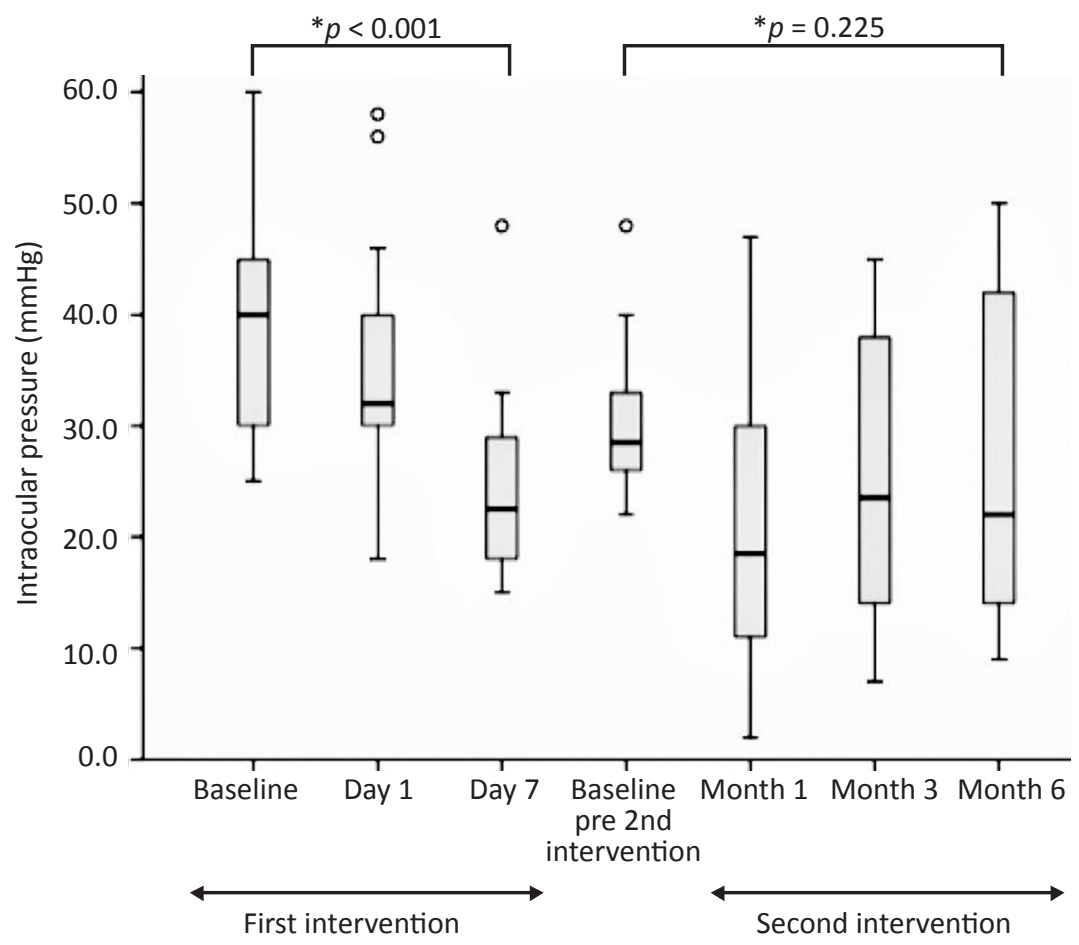

\begin{tabular}{cccccccc}
\hline Time & Baseline & Day 1 & Day 7 & Baseline pre 2nd intervention & Month 1 & Month 3 & Month 6 \\
\hline Mean (SD) IOP (mmHg) & $39.0(10.2)$ & $34.6(0.57)$ & $24.4(8.0)$ & $30.4(6.7)$ & $21.5(12.0)$ & $25.4(2.8)$ & $27.0(15.0)$ \\
\hline
\end{tabular}

Figure 3. Intraocular pressure (IOP) profile from baseline and after the first intervention using intravitreal bevacizumab (IVB) combined with panretinal photocoagulation (PRP) laser, followed by a second intervention (Ahmed implant surgery and cyclodestructive) in all eyes

in month-1, 25.4 (12.8) mmHg in month-3, and 27 (15) mmHg in month-6 $(p=0.225)$ (Figure 3). However, the final VA ranged from 0.4 to 2.5 (logMAR), which indicated that most of the patients remained blind.

In Ahmed implant subgroup analysis (Figure 4) the mean (SD) of baseline IOP before Ahmed implant intervention was 27.5 (3.2) $\mathrm{mmHg}(25-33 \mathrm{mmHg})$. After Ahmed implantation, 8 eyes out of 14 had IOP less than $18 \mathrm{mmHg}$; at 6-month follow-up, there was a $33 \%$ decrease from the initial IOP, with a mean (SD) of IOP was 14 (1.2) mmHg. However, in 14 eyes, the mean (SD) of final IOP was 25 (14.2) $\mathrm{mmHg}$, showing 


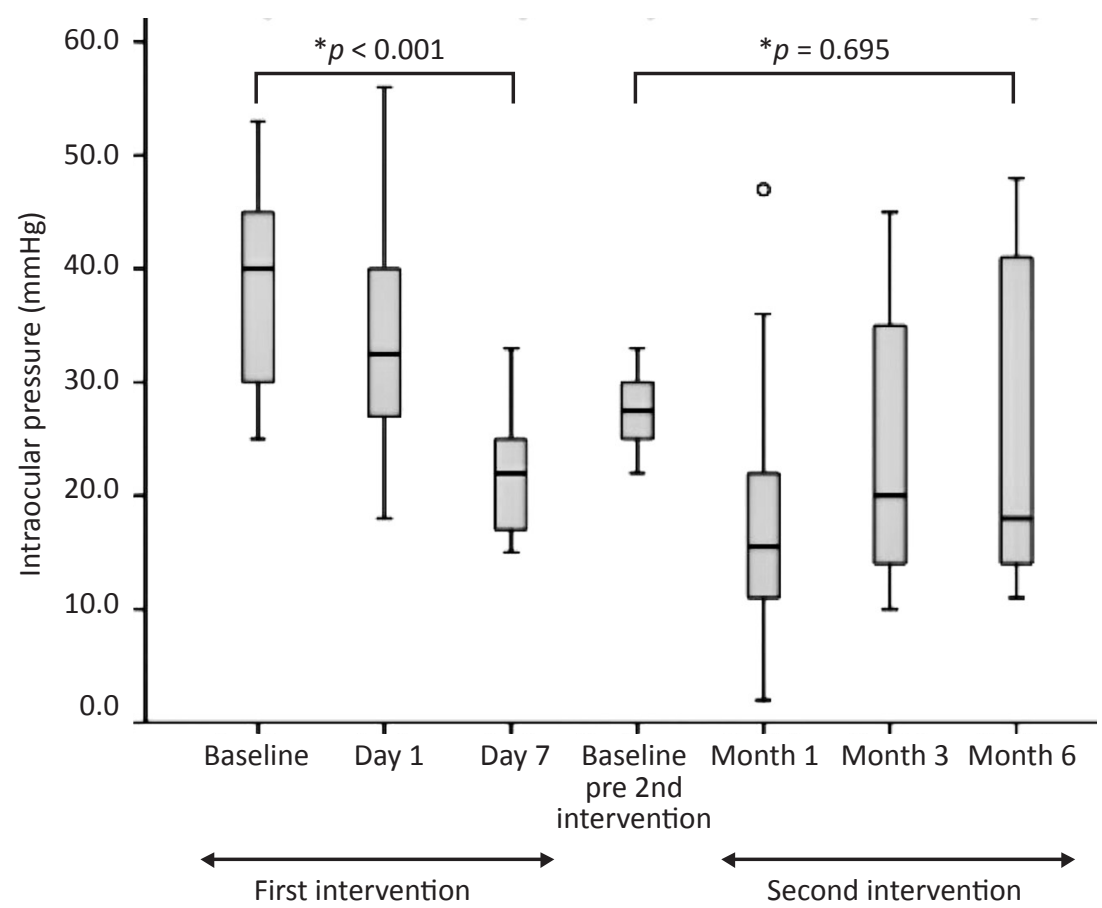

\begin{tabular}{cccccccc}
\hline Time & Baseline & Day 1 & Day 7 & Baseline pre 2nd intervention & Month 1 & Month 3 & Month 6 \\
\hline Mean (SD) IOP (mmHg) & $38.1(9.5)$ & $33.5(10.0)$ & $21.9(5.4)$ & $27.5(3.2)$ & $18.2(11.4)$ & $24.1(12.2)$ & $25.0(14.2)$ \\
\hline
\end{tabular}

Figure 4. Intraocular pressure (IOP) profile from baseline and after the first intervention using intravitreal bevacizumab (IVB) combined with panretinal photocoagulation (PRP) laser followed by Ahmed implant surgery in 14 eyes

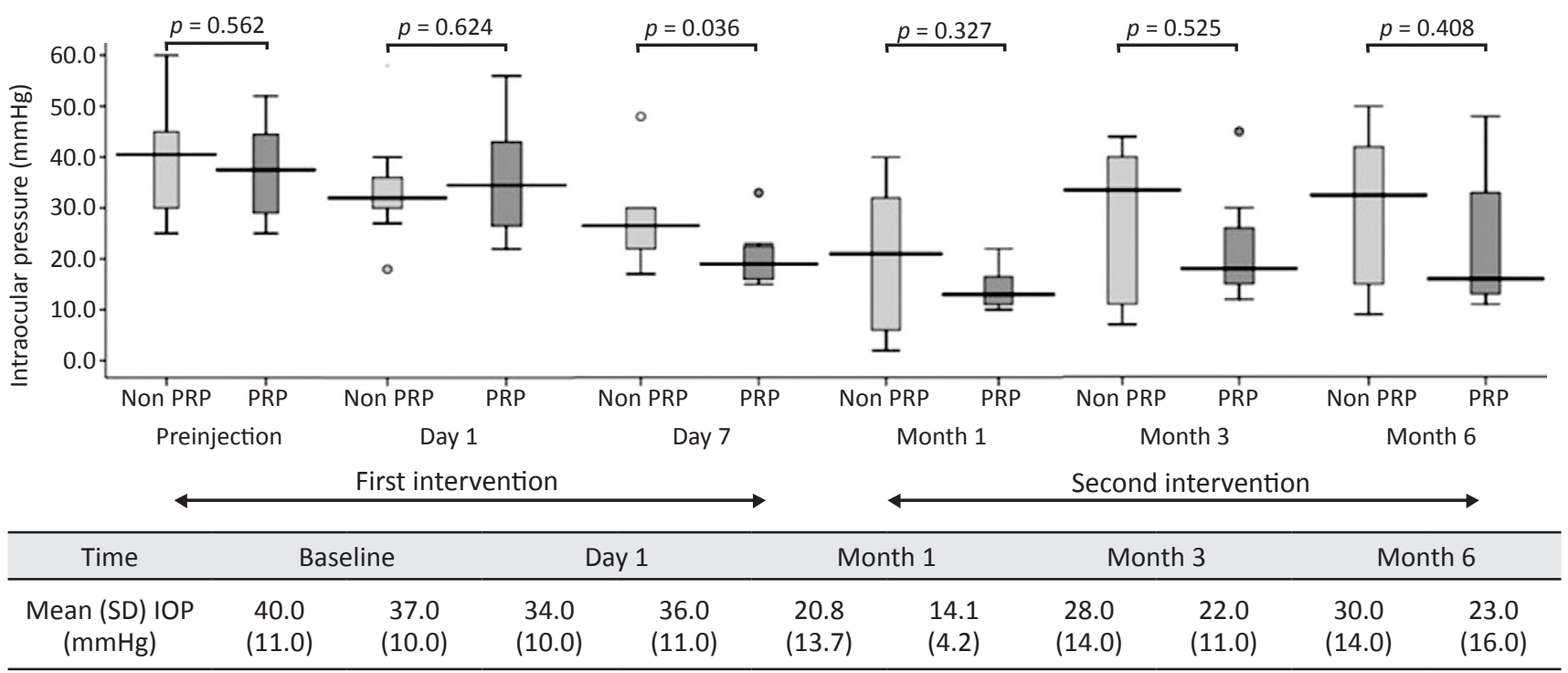

Figure 5. Subgroup analysis between panretinal photocoagulation (PRP) and non-PRP eyes over the follow-up period

no significant difference when compared with baseline IOP of the second intervention ( $p=0.695)$. In terms of VA, VA in 7 eyes remained unchanged (counting fingers <3/60), VA in 5 eyes became worse (light perception), 1 eye with VA showed improvement from 1 to $0.1 \log M A R$ (Aqueous level of $\mathrm{VEGF}=1,688 \mathrm{pg} / \mathrm{dl}$ ), and 1 eye with $\mathrm{VA}$ from 0.4 into $0.1 \log M A R$ (Aqueous level of VEGF = 2,711 pg/ dl) after phacoemulsification surgery. In the end, VA was improved in only 2 eyes, and two additional IVB injections and one additional PRP laser treatment were required during follow-up for the management of new emerging NVI.

Figure 5 shows subgroup analysis of PRP and non-PRP eyes with respect to IOP prior to IVB given during the study period. In the subgroup with 
previous PRP, the level of VEGF appeared low but was unaffected by the decrease in IOP; only in month-1 after the intervention was a significant improvement observed. A similar reduction in IOP was revealed in the non-PRP subgroup as well.

\section{DISCUSSION}

The primary goal of NVG treatment is to reduce IOP and posterior segment ischemia immediately, as well as to treat the underlying cause in order to prevent permanent blindness.7,13,17 Treatment for NVG is still far from satisfactory, mostly due to the primary underlying disease, uncontrolled diabetes, which induces an uncertain hypoxia-ischemia profile. The IOP can be lowered through a combination of procedures, for example, administering antiglaucomatous medications together with PRP laser treatment as a first line of management, followed by glaucoma surgery (trabeculectomy, glaucoma drainage implant surgery, and glaucoma microstent) with or without intravitreal anti-VEGF injection when the IOP is above normal or paracentesis for rapid lowering IOP as a first intervention, followed by PRP laser treatment as a mainstay therapy to control neovascularization. ${ }^{13-15,17,18}$

Laser treatment is the most common basic treatment for NVG, the aim of which is to reduce hypoxia in the surrounding retina and to recover the homeostatic balance between pro-angiogenic (i.e., VEGF) and anti-angiogenic factors. Moreover, PRP laser treatment effectively improves the state of retinal blood circulation and inhibits further release of VEGF. Laser PRP still has a positive effect, as indicated in this study by the lower levels of VEGF in the PRP subgroup compared with the non-PRP subgroup.

Despite the great benefits of PRP laser intervention in managing NVG, this form of treatment cannot be applied in all cases, particularly in those with corneal and vitreous haziness or cataracts. ${ }^{7,8,15,17}$ Furthermore, most traditional surgical interventions for glaucoma do not effectively reduce IOP due to the large number of neovessels on the iris surface and the angle. Damage to these vessels can lead to intraoperative bleeding.7,13,19 Therefore, the use of fast-acting anti-VEGF agents is rationalized prior to such surgery in order to reduce and induce anterior segment neovascularization regression. ${ }^{20,21}$
Anti-VEGFinduceregression of neovascularization in PDR and also facilitate rapid regression of anterior segment neovascularization. 13,14,17-23 Several studies have reported promising results from the use of IVB injection to reduce $\mathrm{NVI}$ and the anterior chamber angle within a short period (2-4 days).7,15 This study showed that regression of NVI occurred in most subjects within a week following IVB injection combined with PRP laser. Therefore, IVB injection at a dose of $1.25 \mathrm{mg}$ should be expected to bring about effective results to eliminate NVG while reducing the IOP. However, in this study, it appears that there was only slight improvement and mild reduction in IOP after the first procedure, even following PRP laser treatment. Due to the remaining increase in IOP after 1 month despite the use of anti-glaucoma eye drops, all eyes still required glaucoma surgery to prevent further optic nerve damage or to save the cornea from bullous keratopathy and eye pain. The shortlived effect of IVB injection combined with PRP laser treatment is thought to be a result of the limited effect of the anti-VEGF agent, which only reduces neovascularization but cannot restore the function of the cicatrized angle or correct retinal hypoxia, as well as incurring irreversible optic nerve damage, which affects the patient's vision. Moreover, the advanced stage of NVG in all patients was also a significant factor that influenced the final outcome of this study. However, this study emphasizes that anti-VEGF treatment is an important first step in the management strategy for NVG.

Reduction of the high baseline IOP was found to be statistically significant within 1 week after combined IVB-PRP laser treatment. Nevertheless, the mean (SD)of final IOP reduction was only approximately 24.4 (8.0) $\mathrm{mmHg}$, with maximal glaucoma medication, still above the IOP target, but then the IOP was increased gradually. It is assumed that optimal IOP reduction may occur only if the residual angle is still open and retains its filtration function. Consequently, if peripheral anterior synechiae in the angle have developed around the trabecular meshwork in all quadrants, the angle will remain permanently closed, and lowering IOP may no longer bring about any beneficial effect. On the other hand, when only a small degree of anterior synechiae occurs, there is a possibility for the anatomic angle to be reversibly opened due to regression of a slight neovascularization membrane. 
If the trabecular meshwork is still visible and healthy, IVB injection will be sufficient to prevent development of primary angle closure. ${ }^{19-22}$ In the early stages of NVG pathology, the angle chamber is still open; however, if not handled in the correct way, the anterior chamber angle will close due to neovascularization, connective tissue membrane and fibrosis. This mechanism occurs along with increasing high cytokines to induce an inflammatory reaction and will cause damage to the trabecular meshwork structures. ${ }^{5}$

In this study, the mean aqueous humor VEGF level in subjects with NVG eyes was comparable to that in other studies, as was the correlation between IVB injection and reduction of the degree of NVI. ${ }^{24-27}$ The range of VEGF levels in this study, however, was wide. Factors that might contribute to this finding include the severity of inflammation and duration of illness, an advanced stage of NVG, improper delivery of PRP laser treatment, and poor diabetic control among patients. Despite the Iow aqueous humor VEGF level, some cases still presented with high IOP and positive NVI. It seems that the appearance of rubeosis iris is due to many factors, the main one being high glucose levels, which greatly affect the overall retinal environment. It is assumed that inflammatory growth factors, such as transforming growth factor $\beta 2$, epidermal growth factor, and others, may influence such conditions, indicating that VEGF might not be the only factor. It is imperative that further studies be carried out for clarification. Furthermore, there was a positive correlation between VEGF concentration and IOP: the higher the VEGF concentration, the higher the IOP. However, this is not in line with the final IOP results of anti-VEGF administration, which were not significantly different between both subgroups and the entire study population. Intravitreal anti-VEGF injections were shown to result in a temporary IOP decrease; as mentioned above, other factors may contribute to IOP regulation in the study subjects, including damage to woven trabecular meshwork, anterior peripheral synechiae, and other anterior tissue structural damage for uveal outflow.

Even though VA was not stated as the primary outcome of this study, there were 16 eyes showed no change or even worsening of final VA. Only 2 eyes showed significant visual improvement. This finding might be related to the severity of existing optic neuropathy due to persistent high IOP, the ischemic nature of the disease, and inappropriate treatment for diabetic retinopathy over an extended period of time. Similar results have also been demonstrated in other studies showing unchanged final VA in patients with initial poor VA (counting fingers and light perception). ${ }^{17,19}$ In contrast, another study reported better results in eyes with early NVI. 15,20

From this study, on management patient with DM and NVG eyes in the Indonesian population: the first step in the controlling of NVG is to reduce IOP as quickly as possible in order to minimize optic nerve damage caused by a high IOP. If NVG is recent and not severe, paracentesis can be considered as an appropriate initial rapid and safe treatment but should be accompanied with anti-VEGF injections to eliminate neovascularization and to prevent bleeding during subsequent glaucoma surgery. AntiVEGF has also been proven to have the beneficial effect of making the retina clearer for PRP to be conducted. Close follow-up and monitoring of IOP should be performed until the IOP decreases to a normal level, and IOP should be kept stable below $18 \mathrm{mmHg}$ throughout the treatment process. As PRP is a fundamental treatment for ischemia retinal disease, it is an essential procedural next step.

This study has shown that all eyes required glaucoma surgery and, in most cases, a glaucoma implant, an obvious indication that all patients who come to a tertiary hospital are already in an advanced stage. Therefore, more intensive management for these NVG patients is strongly recommended. In other words, to perform glaucoma drainage implant surgery along with IVB injection as an adjunct to provide better and more efficient results, the procedure should also be followed by PRP.? Generally, the choice of this sequential management by a glaucoma specialist is rewarded by long-term results. ${ }^{13,15}$ More importantly, it is imperative that regular follow-up of NVG patients is undertaken throughout the treatment process, including stabilization of the blood glucose level.

This study had limitations, including the small sample size, subject characteristics, inclusion of patients with previous PRP laser intervention, and short duration of follow-up. In addition, indocyanine green angiography to determine the exact quantification of NVI were not performed. ${ }^{27}$ However, this study emphasizes that the IOP was 
still above normal limits even after paracentesis followed by IVB injection combined with PRP laser treatment. As a result, glaucoma surgery had to be performed in all of our subjects. Therefore, gonioscopy examination is crucial to assess the stage of NVG, thus ensuring careful and thorough NVG management. The evidence suggests that IVB injection of an anti-VEGF agent combined with PRP laser treatment may be particularly beneficial for early-onset elevated IOP prior to glaucoma surgery to prevent intraoperative bleeding. ${ }^{15,28,29}$ Further glaucoma surgery might be selected, not only with a glaucoma drainage device, which is expensive, but the potency of a trabeculectomy with mitomycin $C$ should be well-thought-out. ${ }^{19}$ However, a carefully considered treatment plan must be established according to the individual characteristics of NVG. ${ }^{30}$

In conclusion, treatment with intravitreal antiVEGF injection combined with PRP was effective in short-term reduction of IOP and NVI regression; however, severe stages of NVI are assumed to be causative of a second increase in IOP, necessitating aggressive management in these population.

\section{Conflict of Interest}

The authors affirm no conflict of interest in this study.

\section{Acknowledgment}

None.

\section{Funding Sources}

None.

\section{REFERENCES}

1. Wild S, Roglic G, Green A, Sicree R, King H. Global prevalence of diabetes: estimates for the year 2000 and projections for 2030. Diabetes Care. 2004;27(5):1047-53.

2. Aiello LP, Northrup JM, Keyt BA, Takagi H, Iwamoto MA. Hypoxic regulation of vascular endothelial growth factor in retinal cells. Arch Ophthalmol. 1995;113(12):1538-44.

3. Aiello LP, Avery RL, Arrigg PG, Keyt BA, Jampel HD, Shah ST, et al. Vascular endothelial growth factor in ocular fluid of patients with diabetic retinopathy and other retinal disorders. N Engl J Med. 1994;331(22):1480-7.

4. Ferrara N. Vascular endothelial growth factor: basic science and clinical progress. Endocr Rev. 2004;25(4):581-611.

5. Davidorf FH, Mouser JG, Derick RJ. Rapid improvement of rubeosis iridis from a single bevacizumab (Avastin) injection. Retina. 2006;26(3):354-6.

6. Malik RA, Li C, Aziz W, Olson JA, Vohra A, McHardy KC, et al. Elevated plasma CD105 and vitreous VEGF levels in diabetic retinopathy. J Cell Mol Med. 2005;9(3):692-7.

7. Rodrigues GB, Abe RY, Zangalli C, Sodre SL, Donini FA, Costa DC, et al. Neovascular glaucoma: a review. Int J Retin Vitr. 2016;2:26.

8. Al-Bahlal A, Khandekar R, Al Rubaie K, Alzahim T, Edward DP, Kozak I. Changing epidemiology of neovascular glaucoma from 2002 to 2012 at King Khaled Eye Specialist Hospital, Saudi Arabia. Indian J Ophthalmol. 2017;65(10):969-73.

9. Hayreh SS. Neovascular glaucoma. Prog Retin Eye Res. 2007;26(5):470-85.

10. Lazcano-Gomez G, R Soohoo J, Lynch A, N Bonell L, Martinez K, Turati $M$, et al. Neovascular glaucoma: a retrospective review from a Tertiary Eye Care Center in Mexico. J Curr Glaucoma Pract. 2017;11(2):48-51.

11. Tripathi RC, Li J, Tripathi BJ, Chalam KV, Adamis AP. Increased level of vascular endothelial growth factor in aqueous humor of patients with neovascular glaucoma. Ophthalmology. 1998;105(2):232-7.

12. Reina-Torres E, Wen JC, Liu KC, Li G, Sherwood JM, Chang JYH, et al. VEGF as a paracrine regulator of conventional outflow facility. Invest Ophthalmol Vis Sci. 2017;58(3):1899-908.

13. Venkat A, Singh R, Eisengart J, Ming H, Ehlers J, Babiuch A. Neovascular glaucoma management: practice patterns of glaucoma and retina specialists in the United States. Invest Ophthalmol Vis Sci. 2017;58(8):3668.

14. SooHoo JR, Seibold LK, Kahook MY. Recent advances in the management of neovascular glaucoma. Semin Ophthalmol. 2013;28(3):165-72.

15. Simha A, Braganza A, Abraham L, Samuel P, Lindsley K. Antivascular endothelial growth factor for neovascular glaucoma. Cochrane Database Syst Rev. 2013;(10):CDo07920.

16. Allingham R, Damji K, Freedman S, Moroi S, Shafranov G. Shields Textbook of Glaucoma 5th Edition. Philadelphia: Lippincott Williams \& Wilkins; 2005. p. 27-512.

17. Sun $Y$, Liang $Y$, Zhou $P$, Wu H, Hou $X$, Ren $Z$, et al. AntiVEGF treatment is the key strategy for neovascular glaucoma management in the short term. BMC Ophthalmol. 2016;16(1):150.

18. Olmos LC, Lee RK. Medical and surgical treatment of neovascular glaucoma. Int Ophthalmol Clin. 2011;51(3):27-36.

19. Higashide T, Ohkubo S, Sugiyama K. Long-term outcomes and prognostic factors of trabeculectomy following intraocular bevacizumab injection for neovascular glaucoma. PLoS One. 2015;10(8):e0135766.

20. Campochiaro PA, Aiello LP, Rosenfeld PJ. Anti-vascular endothelial growth factor agents in the treatment of retinal disease: from bench to bedside. Ophthalmology. 2016;123(10S):S78-88.

21. Costagliola C, Cipollone U, Rinaldi M, della Corte M, Semeraro F, Romano MR. Intravitreal bevacizumab (Avastin ${ }^{\circledR}$ ) injection for neovascular glaucoma: a survey on 23 cases throughout 12-month follow-up. Br J Clin Pharmacol. 2008;66(5):667-73.

22. Wu L, Martinez-Castellanos MA, Quiroz-Mercado H, Arevalo JF, Berrocal MH, Farah ME, et al. Twelve-month safety of intravitreal injections of bevacizumab (Avastin): results of the Pan-American Collaborative Retina Study Group (PACORES). Graefes Arch Clin Exp Ophthalmol. 2008;246(1):81-7.

23. Ichhpujani P, Ramasubramanian A, Kaushik S, Pandav SS. Bevacizumab in glaucoma: a review. Can J Ophthalmol. 2007;42(6):812-5.

24. Beutel J, Peters S, Luke M, Aisenbrey S, Szurman P, Spitzer MS, et al. Bevacizumab as adjuvant for neovascular glaucoma. Acta Ophthalmol. 2010;88(1):103-9.

25. Matsuyama K, Ogata N, Jo N, Shima C, Matsuoka M, Matsumura $M$. Levels of vascular endothelial growth factor and pigment epithelium-derived factor in eyes before and after intravitreal injection of bevacizumab. Jpn J Ophthalmol. 2009;53(3):243-8.

26. Lim TH, Bae SH, Cho YJ, Lee JH, Kim HK, Sohn YH. Concentration of vascular endothelial growth factor after intracameral bevacizumab injection in eyes with neovascular glaucoma. Korean J Ophthalmol. 2009;23(3):188-92.

27. Blum A, Socea D, Ben-Shushan RS, Keinan-Boker L, Naftali M, Segol G, et al. A decrease in VEGF and inflammatory markers is associated with diabetic proliferative retinopathy. Eur Cytokine Netw. 2012;23(4):158-62. 
28. Li ZQ, Zhou XX, Lin S, Li JL, Wu JG. Angiography reveals early hiding iris neovascularization after ischemic CRVO. Int J Ophthalmol. 2013;6(2):253-4.

29. Tang $M$, Fu $Y$, Wang $Y$, Zheng $Z$, Fan $Y$, Sun $X$, et al. Efficacy of intravitreal ranibizumab combined with Ahmed glaucoma valve implantation for the treatment of neovascular glaucoma. BMC Ophthalmol. 2016;16:7.

30. Kernt M, Neubauer AS, Kampik A. Intravitreal bevacizumab (Avastin) treatment is safe in terms of intraocular and blood pressure. Acta Ophthalmol Scand. 2007;85(1):119-20. 